\title{
A POWERFUL DISINFECTANT
}

OptiZil is a powerful disinfectant that helps dental practices clean surfaces up to five times faster than products with a five-minute contact time.

The non-toxic sanitiser is proven effective against $99.9999 \%$ of bacteria and viruses in '1-minute' and approved for BS EN14476 (virucidal test).

OptiZil multi-surface disinfectant is part of the CleanCert Innovations $360^{\circ}$ - approach to effective clinical infection control. The range also includes CleanCert waterline cleaner and biofilm remover and OraWize+ antimicrobial mouthwash.

The active ingredient in every product is Hypochlorous acid ( $\mathrm{HOCl}$ ), a natural biocide that is exceptionally potent yet non-toxic, non-carcinogenic, or a mucosa irritant.

Thanks to an innovative manufacturing process, the Hypochlorous in OptiZil, CleanCert and OraWize+ is stabilised for 12-months shelf life and produced at neutral $\mathrm{pH} 7$.

Four key benefits of using OptiZil:

1. Fast-acting. The oneminute contact time helps turnaround dental stations and environments much quicker in the fallow time

2. No pre-mixing required. Staff save time because our products are pre-mixed and ready to use straight from the container

3. No need to spend time on staff training. No need to train staff in hazardous chemical use as OptiZil, CleanCert and OraWize+ are biocompatible and nontoxic

4. Benefit from bulk-buy discounts. The 12-month shelf life means practices can buy in bulk to save money without worrying about products losing efficacy.

Ask your distributor about special offers. Or contact sales@cleancert.co.uk.

\section{YOUR FIRST LINE OF DEFENCE}

Are you worried about what to do should you have a suspected case of Coronavirus in your aesthetic clinic? Put your fears at ease with the new Infection Containment Kit from Initial Medical, designed for touchpoint cleaning and the disinfection of potentially contaminated surfaces.

Containing PPE, specialist Steri-7 infection control products, hand sanitiser, workplace barrier tape and a rigid container for waste disposal, this kit is the best first response you can have to hand for a suspected infection onsite. It comes with step-by-step guidelines and once used the waste will be collected and disposed of responsibly by the Initial Medical team.

Suitable to help contain a wide range of suspected infections including Coronavirus, Norovirus and MRSA, it's the perfect solution to have on hand. In the event of a confirmed case of Coronavirus onsite then a specialist disinfection service should be undertaken by professionals such as Rentokil Specialist Hygiene who are equipped with the relevant the correct personal protective equipment (PPE), respiratory protective equipment (RPE) and specialist disinfection equipment.

Visit www.initial.co.uk/medical or call 08708504045 .

\section{NEW MACHINES OFFER UNBEATABLE HYGIENE STANDARDS}

Miele's latest range of professional washing machines and dryers set new standards for the hygienic laundering of dental scrubs and protective uniforms.

The Little Giants range comprises the Performance and Performance Plus washing machines and dryers, which offer powerful on-site washing for peace of mind regarding infection control.

The machines fulfil Government guidelines for on-site laundry facilities to clean workwear, with the added benefits an 85-degree thermal disinfection programme, as well as short wash cycles, a convenient stack footprint of less than $1 \mathrm{~m}^{2}$ and a range of thoughtful, labour-reducing features.

The commercial grade machines with Wi-Fi connectivity feature Miele's ecofriendliest professional laundry technology yet, with cycle times of only 49 minutes for washing and 38 minutes for drying. The new heat-pump dryer has outperformed its predecessor by one third, with a cycle time of less than 60 minutes, using $60 \%$ less energy than a standard condenser dryer. Washers feature Miele's patented honeycomb drum for ultimate fabric care.

Due to the speed capacity of washing

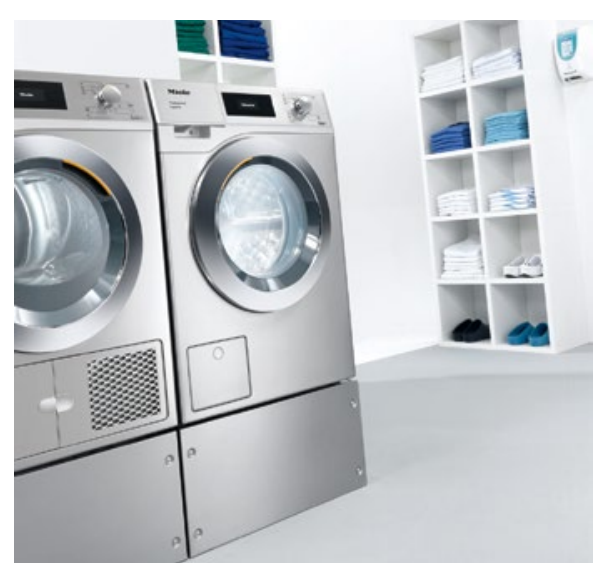

cycles, the new range washing machines require $22 \%$ less water and $28 \%$ less energy. While casing dimensions have remained unchanged, washing machines and tumble dryers now have a load capacity increased by $25 \%$.

The Performance washing machines have been given an $\mathrm{A}+++$ energy efficiency rating, while the Performance heat-pump dryer has been awarded A++. Adding to their sustainable credentials, $95 \%$ of the machines can be recycled at end of life.

The high performance machines complement Miele's specialist washerdisinfectors, which provide dependable, validated cleaning of dental instruments.

For more information or to order email lab@miele.co.uk or visit the Miele GB website: https://www.miele.co.uk/ professional/new-little-giants-2009. htm\#p2012. 